\title{
Low complexity sparse beamspace DOA estimation via single measurement vectors for uniform circular array
}

\author{
Di Zhao ${ }^{1,2^{*}}$ (D), Weijie Tan ${ }^{3}$, Zhongliang Deng ${ }^{1}$ and Gang Li
}

\author{
${ }^{*}$ Correspondence: \\ zhaodi820@bupt.edu.cn \\ 'Wireless Network Positioning and \\ Communication Integration \\ Research Center, School of \\ Electronic Engineering, Beijing \\ University of Posts and \\ Telecommunications, 100876 \\ Beijing, China \\ ${ }^{2}$ The 54th Research Institute of \\ CETC, 050081 Shijiazhuang, China \\ Full list of author information is \\ available at the end of the article
}

\begin{abstract}
In this paper, we present a low complexity sparse beamspace direction-of-arrival (DOA) estimation method for uniform circular array (UCA). In the proposed method, we firstly use the beamspace transformation (BT) to transform the signal model of UCA in element-space domain to that of virtual uniform linear array (ULA) in beamspace domain. Subsequently, by applying the vectoring operator on the virtual ULA-like array signal model, a novel dimension-reduction sparse beamspace signal model is derived based on Khatri-Rao (KR) product, the observation data of which is represented by the single measurement vectors (SMVs) via vectorization of sparse covariance matrix. And then, the DOA estimation is formulated as a convex optimization problem by following the concept of a sparse-signal-representation (SSR) of the SMVs. Finally, simulations are carried out to validate the effectiveness of the proposed method. The results show that without knowledge of the number of signals, the proposed method not only has higher DOA resolution than the subspace-based methods in low signal-to-noise ratio (SNR), but also has far lower computational complexity than other sparse-like DOA estimation methods.
\end{abstract}

Keywords: Direction-of-arrival estimation, Uniform circular array, Low complexity, Beamspace transformation, Convex optimization

\section{Introduction}

In the past decades, direction-of-arrival (DOA) estimation of propagating plane waves for uniform circular array (UCA) has been widely used in various fields, such as communication, radar, sonar, radio astronomy and so on [1]. The DOA estimation methods and their derivatives are divided into three categories: beamforming techniques [2, 3], subspace-based methods [4, 5], and the maximum likelihood approach [6, 7]. Besides these approaches, sparse-signal-representation (SSR)-based DOA estimation methods [8-12] have been paid great attention in recent years, which are widely used in elementspace domain, beamspace domain [13], and various scenarios where mixture (coherent and incoherent, or circular and non-circular $[14,15])$ signals exist as well. They are different from the conventional representative methods, such as the Capon beamformer [16]

(c) The Author(s). 2021 Open Access This article is licensed under a Creative Commons Attribution 4.0 International License, which permits use, sharing, adaptation, distribution and reproduction in any medium or format, as long as you give appropriate credit to the original author(s) and the source, provide a link to the Creative Commons licence, and indicate if changes were made. The images or other third party material in this article are included in the article's Creative Commons licence, unless indicated otherwise in a credit line to the material. If material is not included in the article's Creative Commons licence and your intended use is not permitted by statutory regulation or exceeds the permitted use, you will need to obtain permission directly from the copyright holder. To view a copy of this licence, visit http://creativecommons.org/licenses/by/4.0/. 
and the multiple signal classification (MUSIC) $[17,18]$. In conventional DOA methods, the observation data matrix is generally treated as a linear combination of the steering vectors and incoming signals plus stochastic noise [19]. However, the observation data matrix of the SSR-based DOA estimation approaches is formulated by the sparselyrepresented signal vectors and the overcomplete basis, where the overcomplete basis is comprised of spatial samples from the array manifold [10] on the premise that the DOAs of signals fall sparsely into the entire spatial domain. In [8], the $\ell_{1}$-SRACV is based on a sparse representation of array covariance vectors and applies the sparsity constraints to an $\ell_{1}$-norm minimization problem for improving the DOA estimation performance. In [10], $\ell_{1}$-SVD sparsely represents the signal subspace by the singular value decomposition (SVD). In [9], a low complexity sparse covariance-based DOA estimation method called LC-SRACV is proposed, which uses the Khatri-Rao (KR) product in SSR framework to recover array covariance vectors of only one single measurement vector.

These methods mentioned above are all manipulated in element-space domain. In beamspace domain, the beamspace transform (BT) technique $[20,21]$ is mainly adopted to solve the DOA estimation problem of UCA. It is a kind of manifold separation technique [22] that is suitable for an arbitrary array. It substantially is a modal transform that maps the steering vectors of a UCA to that of a virtual uniform linear array (ULA) with Vandermonde structure. Using the technique, some DOA estimators with MUSIC in beamspace domain and derives, such as Real Beamspace MUSIC (RB-MUSIC)[17] and unitary ESPRIT in beamspace domain [23], are proposed. However, the estimators rely heavily on a priori known signal number, which has a close relationship with noise subspace, and have less capability of angular separation if the number of sources beyond the number of the sensors.

In this paper, we propose a low complexity sparse beamspace DOA estimation for UCA by vectorizing the array covariance vectors, called BS- $\ell_{1}$-SRSMVS, which exploits the methodology combining the BT technique and the SSR model of single measurement vectors (SMVs) in beamspace domain. Having transformed the signal model from UCA to virtual ULA and vectorized the observation data, a KR-based virtual ULA signal model [24] is proposed. The new array manifold matrix of the signal model can be decomposed into the product of a selection matrix and a Vandermonde vector. Comparing the subspace-based method as RB-MUSIC, the proposed method does not need a priori knowledge of the number of sources; meanwhile, it has higher performance of angle resolution. Due to the centro-Hermitian characteristics of the steering vectors of virtual ULA, using Khatri-Rao product, the sparse vectors to be estimated can be recovered with single measurement vectors rather than multiple measurement vectors (MMVs) [25, 26] used in other SSR-based DOA estimation methods, such as $\ell_{1}$-SVD and $\ell_{1}$-SRACV. The difficulty in SSR-based DOA estimation for UCA is that how to reduce dimensionality of the observation data for lower computational complexity. The kernel ideology of the new methodology is that transforming the observation data of UCA to that of virtual ULA, which is a prerequisite for the downscaling of the observed data, and applying the vectorization to the covariance observation data of virtual ULA, which makes the covariance matrix reduced to the SMVs.

This paper is organized as follows. In Section 2, we overview the related works of SSRbased DOA estimation methods. In Section 3, we derive the steering vectors of the virtual ULA in beamspace domain via the BT technique and induce a dimension-reduction vir- 
tual array signal model for UCA. Subsequently, we propose a new sparse beamspace DOA estimation method with SMV version. In Section 4, we analyse the computational complexity of different methods theoretically. In Section 5, simulation experiments are conducted to demonstrate the performance of the proposed method. Finally, Section 6 concludes the paper.

\section{Related works}

Here we focus on some DOA estimation methods based on sparse signal representation $[8-10,15]$. The models are reconstructed using types of vectors, which are introduced in Sections 2.1 and 2.2. The advantages and disadvantages of these methods are introduced in Section 2.3.

\subsection{Singular vectors of observation data matrix}

In this case, the observation data matrix is generally processed in element-space domain. Using the singular value decomposition transformation [10], a signal model composed of $K$ singular vectors is reconstructed, namely the singular vectors space. The method called $\ell_{1}$-SVD. Its signal model is formulated as $\mathbf{Y}^{\mathrm{sv}}=\mathbf{A} \mathbf{S}^{\mathrm{sv}}+\mathbf{N}^{\mathrm{sv}}$, where the superscript of $(\bullet)^{\mathrm{sv}}$ denotes the operation of SVD $, \mathbf{Y}^{\mathrm{sv}}, \mathbf{A}, \mathbf{S}^{\mathrm{sv}}$ and $\mathbf{N}^{\mathrm{sv}}$ are respectively observation data matrix of signals, array manifold matrix, impinging signals and noise. $\mathbf{S}^{\text {sv }}$ is a twodimension matrix, which is indexed by rows with spatial angle samples whose dimension ranges from 1 to $Q$ and by columns with singular vectors whose dimension ranges from 1 to $K$. Thus the $\ell_{2}$-norm of the $i$-th row vector $\mathbf{s}_{i}$ of $\mathbf{S}^{\mathrm{sv}}$ corresponds to the sparsity of the spatial spectrum, where $\left\|\mathbf{s}_{i}\right\|_{2}=\sqrt{\sum_{k=1}^{K} s_{i}(k)^{2}}, k=1, \cdots, K, s_{i}(k)$ is the $k$-th element of $\mathbf{s}_{i}, i=1, \ldots, Q, Q$ is the number of spatial angle samples. Here it is simplified to $\mathbf{s}_{i}^{\left(\ell_{2}\right)}$. So the DOA estimation is converted to the problem of searching the spatial spectrum of $\mathbf{s}$ by minimizing $\left\|\mathbf{Y}^{\mathrm{sv}}-\mathbf{A} \mathbf{S}^{\mathrm{sv}}\right\|_{\mathrm{F}}^{2}+\lambda\|\mathbf{s}\|_{1}$, where $\mathbf{s}=\left[\mathbf{s}_{1}^{\left(\ell_{2}\right)}, \cdots, \mathbf{s}_{Q}^{\left(\ell_{2}\right)}\right], \lambda$ is the weighted parameter, $\|\bullet\|_{\mathrm{F}}$ is the Frobenius norm. It is obvious that the SVD transformation and $\ell_{2}$ norm of the row-indexed singular vectors are necessary. Generally, using the optimization toolbox, the DOA estimation is transformed to

$$
\min \|\mathbf{s}\|_{1} \text { subject to }\left\|\mathbf{Y}^{\mathrm{sv}}-\mathbf{A} \mathbf{S}^{\mathrm{sv}}\right\|_{\mathrm{F}}^{2} \leq \beta^{2} .
$$

Where $\beta$ is the regularization parameter, however, the choice of which is still an open problem if no knowledge of sources is available.

\subsection{Covariance matrix vectors of signal space}

Another signal representation is the vectorized signal model based on the covariance matrix vectors. As the method introduced in [8], the signal model of $\ell_{1}-\mathrm{SRACV}$ is represented as $\mathbf{R}=\widetilde{\mathbf{A}}(\boldsymbol{\phi}) \mathbf{B}+\sigma^{2} \mathbf{I}_{M}$, where $\mathbf{R}$ is the signal covariance matrix, $\boldsymbol{\phi}=$ $\left[\phi_{1}, \phi_{2}, \cdots, \phi_{Q}\right], \widetilde{\mathbf{A}}(\boldsymbol{\phi})=\left[\tilde{\mathbf{a}}\left(\phi_{1}\right), \tilde{\mathbf{a}}\left(\phi_{2}\right), \cdots, \tilde{\mathbf{a}}\left(\phi_{Q}\right)\right]$ is the overcomplete basis constructed according to the array structure, and $Q$ is the number of the overcomplete basis vectors. $\mathbf{I}_{M}$ is an $M \times M$ identity matrix, in which $M$ is the number of sensors, $\sigma^{2}$ is the power of noise. $\mathbf{B} \in \mathbb{C}^{Q \times M}$ is the matrix composed of multiple measurement column vectors, which all share the same sparse structure. The nonzero elements of $\mathbf{B}$ is appeared in the same rows of the column vectors $\left\{\mathbf{b}^{T}\right\}_{1}^{M}$, where $(\bullet)^{T}$ stands for the transpose. Having solved $\ell_{2}$-norm of $\mathbf{B}$ by rows, i.e. $\left\|\mathbf{b}_{i}\right\|_{2}=\sqrt{\sum_{m=1}^{M} b_{i}^{2}(m)}, m=1, \cdots, M, i=1, \cdots, Q$, 
we can obtain the vector of $\mathbf{b}=\left[\mathbf{b}_{1}^{\left(\ell_{2}\right)}, \cdots, \mathbf{b}_{Q}^{\left(\ell_{2}\right)}\right]$, the nonzero element of which corresponds to a certain vector $\tilde{\mathbf{a}}\left(\phi_{i}\right)$ of the overcomplete basis $\tilde{\mathbf{A}}(\boldsymbol{\phi})$. Here $\left\|\mathbf{b}_{i}\right\|_{2}$ is abbreviated to $\mathbf{b}_{i}^{\left(\ell_{2}\right)}$. The problem is expressed as

$$
\min _{\mathbf{B}}\|\mathbf{b}\|_{1} \text { subject to } \mathbf{R}=\widetilde{\mathbf{A}}(\boldsymbol{\phi}) \mathbf{B}+\sigma^{2} \mathbf{I}_{M} .
$$

Having introduced the Lagrange multiplier $\eta$, (2) is transformed to

$$
\min _{\mathbf{B}}\left\|\mathbf{W} \cdot \operatorname{vec}\left[\mathbf{R}-\tilde{\mathbf{A}}(\boldsymbol{\phi}) \mathbf{B}-\sigma^{2} \mathbf{I}_{M}\right]\right\|_{2}^{2}+\eta\|\mathbf{b}\|_{1},
$$

where $\mathbf{W}$ is the weight matrix related to the covariance matrix $\mathbf{R}, \operatorname{vec}(\bullet)$ denotes the vectorization operator.

Specially for a ULA, a derived method called LC-SRACV is presented in [9]. Due to the centro-Hermitian property of column vectors of $\widetilde{\mathbf{A}}(\boldsymbol{\phi})$, having vectorized the covariance matrix of $\mathbf{B},(3)$ is transformed to

$$
\min _{\mathbf{B}}\left\|\mathbf{W} \cdot\left[\operatorname{vec}(\mathbf{R})-\left(\widetilde{\mathbf{A}}^{*}(\boldsymbol{\phi}) \odot \widetilde{\mathbf{A}}(\boldsymbol{\phi})\right) \mathbf{u}-\sigma^{2} \operatorname{vec}\left(\mathbf{I}_{M}\right)\right]\right\|_{2}^{2}+\eta\|\mathbf{u}\|_{1},
$$

where $\odot$ represents the KR product and $(\bullet)^{*}$ denotes complex conjugate. Here $\mathbf{u}$ is the $Q$-sparse vector. Different from [8], the object to be optimized is not a matrix but a vector.

\subsection{Advantages and disadvantages}

Comparing the subspace-based methods, except for not being sensitive to orthogonality of the signal subspace and noise subspace, the SSR-based DOA estimation approaches also have other superiorities.

As known in [9], LC-SRACV extends the array aperture from $M$ (the number of sensors) to $2 M-1$ and increases the degrees of freedom. And it has much less computational cost than that of $\ell_{1}$-SRACV and $\ell_{1}$-SVD. The $\ell_{1}$-SRACV does not concern any knowledge of covariance array, and it is applicable for an arbitrary array. $\ell_{1}-\mathrm{SVD}[10]$ is not dependent on the knowledge of the noise covariance.

However, there are obvious limitations in these SSR-based DOA estimation methods. For LC-SRACV, its main limitation lies in the fact that the steering vectors must be the special Vandermonde structure, and it is not suitable for UCA completely because the elements of overcomplete basis are not only dependent on direction samples, but also related to the array geometry. They have not a clear monadic corresponding relationship with direction samples. $\ell_{1}$-SVD is dependent on the signal subspace singular vectors and noise power. Its computational complexity has a close relationship with the number of incoming signals. In addition, it is challenging to determine the regularization parameters when no knowledge of noise or sources is available. $\ell_{1}$-SRACV adopts $\ell_{1}$ penalty for sparsity and $\ell_{2}$ penalty for each representation coefficient vector. Two-fold iterations make its computational cost higher than that of the formers.

\section{Proposed method}

In this section, we introduce our proposed method. In Section 3.1, we firstly derive the virtual array signal mode in beamspace domain by using beamspace transforming. And in Section 3.2, we introduce the covariance matrix representation by KR-product. In Section 3.3, we introduce the sparse beamspace DOA estimation method via single measurement vector. 


\subsection{Virtual array signal model in beamspace}

Consider an array composed of $M$ sensors located along the circumference of a uniform circular array with radius $r$. There are $P(P<M)$ narrowband uncorrelated signals impinging on the array in the far-field. $\phi=\left\{\phi_{1}, \phi_{2}, \cdots, \phi_{P}\right\}$ is the set of the incident angles of the signals. The observation data is formulated as

$$
\mathbf{x}(t)=\mathbf{A}(\boldsymbol{\phi}) \mathbf{s}(t)+\mathbf{n}(t), \quad t=1, \cdots, N,
$$

where $\mathbf{x}(t)=\left[x_{1}(t), x_{2}(t), \cdots, x_{M}(t)\right]^{T}$ is an $M \times 1$ noise-corrupted snapshot vector. $\mathbf{s}(t)=\left[s_{1}(t), s_{2}(t), \cdots, s_{P}(t)\right]^{T}$ is a $P \times 1$ signal vector, and $\mathbf{n}(t) \in \mathbb{C}^{M}$ is the assumed zero-mean Gaussian white noise. $N$ is the number of snapshots. $\mathbf{A}(\boldsymbol{\phi})=$ $\left[\mathbf{a}\left(\phi_{1}\right), \mathbf{a}\left(\phi_{2}\right), \cdots, \mathbf{a}\left(\phi_{P}\right)\right] \in \mathbb{C}^{M \times P}$ is the array manifold matrix of the UCA, here $\mathbf{a}\left(\phi_{p}\right), p=1, \cdots, P$, are the $M \times 1$ steering vectors. It can be expressed as

$$
\mathbf{a}\left(\phi_{p}\right)=\left[\begin{array}{c}
e^{j \zeta \cos \left(\phi_{p}-\gamma_{1}\right)} \\
e^{j \zeta \cos \left(\phi_{p}-\gamma_{2}\right)} \\
\cdots \\
e^{j \zeta \cos \left(\phi_{p}-\gamma_{M}\right)}
\end{array}\right],
$$

where $j=\sqrt{-1}, \zeta=k r$, and the wavenumber $k=2 \pi / \lambda$ with $\lambda$ being the wavelength of the incident signals. $\gamma_{\mathrm{m}}=2 \pi(m-1) / M, m=1,2, \cdots, M$, are sensors' locations along the circumference of the UCA.

Assume that the signals $\left\{s_{p}(t)\right\}_{p=1}^{P}$ are uncorrelated for different sources, and also independent of $\mathbf{n}(t)$. The covariance matrix of the observation data $\mathbf{x}(t)$ is given by

$$
\mathbf{R}=\mathrm{E}\left[\mathbf{x}(t) \mathbf{x}^{H}(t)\right]=\mathbf{A}(\boldsymbol{\phi}) \mathbf{R}_{\mathrm{s}} \mathbf{A}^{H}(\boldsymbol{\phi})+\sigma_{n}^{2} \mathbf{I}_{M},
$$

where $\mathbf{R}_{\mathrm{s}}=\mathrm{E}\left[\mathbf{s}(t) \mathbf{s}^{H}(t)\right]$ is the signal covariance matrix, whose diagonal elements are $\left\{\sigma_{s p}^{2}\right\}_{p=1}^{P} . \sigma_{n}^{2}$ is the noise power, $\mathrm{E}(\bullet)$ and $(\bullet)^{H}$ are the expectation and the conjugate transpose operator respectively. The signal mode of the UCA can be transformed to that of a ULA-type array by synthesizing the beamspace manifold, which is similar to that of ULA using phase mode excitation of continuous circular aperture[20]. The signal model of the virtual ULA essentially takes discrete spatial sampling of far-field pattern resulting from all harmonics of array excitation (each harmonic means one phase mode, theoretically it ranges from $-\infty$ to $+\infty$. Actually the magnitude of harmonic decays super-exponentially with increasing harmonic order $h$, i.e. $h$-th phase mode. If $h$ is large enough and reach a certain number $H_{e}$, the magnitude is asymptotically approaching zero) by incoming signals over continuous aperture of UCA[27]. The beamspace manifold synthesized by a beamformer $\mathbf{F}_{e}^{H}=\mathbf{C}_{v} \mathbf{V}^{H}[20]$ is given by

$$
\mathbf{a}_{e}\left(\phi_{p}\right)=\mathbf{F}_{e}^{H} \mathbf{a}\left(\phi_{p}\right)=\mathbf{C}_{v} \mathbf{V}^{H} \mathbf{a}\left(\phi_{p}\right) \approx \sqrt{M} \mathbf{J}_{\zeta} \mathbf{d}\left(\phi_{p}\right),
$$

where

$$
\begin{aligned}
& \mathbf{C}_{v}=\operatorname{diag}\left\{j^{-H_{e}}, \ldots, j^{-1}, j^{0}, j^{-1}, \ldots, j^{-H_{e}}\right\}, \\
& \mathbf{V}=\sqrt{M}\left[\mathbf{w}_{-H_{e}}: \cdots: \mathbf{w}_{0}: \cdots: \mathbf{w}_{H_{e}}\right], \\
& \mathbf{J}_{\zeta}=\operatorname{diag}\left[J_{H_{e}}(\zeta), \ldots, J_{1}(\zeta), J_{0}(\zeta), J_{1}(\zeta), \ldots, J_{H_{e}}(\zeta)\right],
\end{aligned}
$$

where $\mathbf{C}_{v}$ and $\mathbf{J}_{\zeta}$ are $\left(2 H_{e}+1\right) \times\left(2 H_{e}+1\right)$ diagonal matrices. $\mathbf{J}_{\zeta}$ is a matrix of Bessel functions. The amplitudes of Bessel-function coefficients on the master diagonal taper 
symmetrically, and the coefficients act as such a function, that is, linking the amplitude of each phase-mode excitation with that of the corresponding far-field phase-mode pattern. The matrix $\mathbf{V}$ is a normalized beamforming weight matrix that excites the array with a finite number of excitation modes. $h \in\left[-H_{e}, H_{e}\right]$ are phase modes that can be excited. Here a rule of thumb for determining $H_{e}$ is given as $H_{e} \approx \zeta$ and $H_{e}$ should satisfy $H_{e}<M / 2$. The vectors of $\left\{\mathbf{w}_{h}\right\}_{h=-H_{e}}^{H_{e}}$ are regarded as the spatial discrete sampling corresponding to the far-field pattern, which are caused by the $h$-th phase mode excitation along the continuous circular aperture. It is defined by

$$
\mathbf{w}_{h}^{H}=\frac{1}{M}\left[e^{j h \gamma_{1}}, e^{j h \gamma_{2}}, \ldots, e^{j h \gamma_{M}}\right] .
$$

From (8), we know that the steering vectors $\mathbf{a}_{e}\left(\phi_{p}\right)$ in beamspace domain can be represented by the vector $\mathbf{d}\left(\phi_{p}\right)$. It is expressed as

$$
\mathbf{d}\left(\phi_{p}\right)=\left[e^{-j H_{e} \phi_{p}}, \ldots, e^{-j \phi}, 1, e^{j \phi}, \ldots, e^{j H_{e} \phi_{p}}\right]^{T} .
$$

Here $\sqrt{M} \mathbf{J}_{\zeta} \mathbf{d}\left(\phi_{p}\right), p=1,2, \ldots, P$, are the ideal steering vectors of the virtual ULA with Vandermonde structure.

The methodology of phase mode excitation-based beamformer offers the operation on transforming observation data in element-space to that of beamspace. For the observation data illustrated in (5), using the methodology, we have $\mathbf{y}(t) \in \mathbb{C}^{M_{e} \times P}$, which is given by

$$
\mathbf{y}(t)=\mathbf{F}_{e}^{H} \mathbf{x}(t)=\sqrt{M} \mathbf{J}_{\zeta} \mathbf{D}(\boldsymbol{\phi}) \mathbf{s}(t)+\mathbf{F}_{e}^{H} \mathbf{n}(t),
$$

here $M_{e}=2 H_{e}+1$ is the total number of excited modes. From (14), we know that the observation data $\mathbf{x}(t)$ of $M \times P$ dimensions in element-space domain is mapped to a dimension-reduction matrix $\mathbf{y}(t)$ of $M_{e} \times P$ dimensions in beamspace domain. And the term $\sqrt{M} \mathbf{J}_{\zeta} \mathbf{D}(\boldsymbol{\phi}) \mathbf{s}(t)$ is a noise-free beamspace data matrix, which is expressed as a product of virtual array manifold $\mathbf{D}(\phi)$, the source vector $\mathbf{s}(t)$ and Bessel functions. Here $\mathbf{D}(\boldsymbol{\phi})=\left[\mathbf{d}\left(\phi_{1}\right), \mathbf{d}\left(\phi_{2}\right), \ldots, \mathbf{d}\left(\phi_{P}\right)\right]$ has centro-Hermitian columns with Vandermonde structure, $\mathbf{F}_{e}^{H}$ is a unitary matrix that satisfies $\mathbf{F}_{e}^{H} \mathbf{F}_{e}=\mathbf{I}_{M_{e}}$. The by-product $\mathbf{F}_{e}^{H} \mathbf{n}(t)$ of the transformation still remains the white Gaussian process. Thus, we have the covariance matrix of the observation data $\mathbf{y}(t)$. It is given by

$$
\mathbf{R}_{\mathbf{y}}=M \mathbf{J}_{\zeta} \mathbf{D}(\phi) \mathbf{R}_{\mathbf{s}} \mathbf{D}^{H}(\phi) \mathbf{J}_{\zeta}+\sigma_{n}^{2} \mathbf{I}_{M_{e}}
$$

\subsection{KR-based covariance matrix representation}

In this subsection, we apply the KR subspace approach to DOA estimation[24] of the virtual ULA. For the signal representation formulated in the above section, applying the vectorization operator on (15), we have a new array model expressed as

$$
\mathbf{Y}:=\operatorname{vec}\left(\mathbf{R}_{\mathbf{y}}\right)=M\left[\left(\mathbf{J}_{\zeta}^{*} \mathbf{D}^{*}(\boldsymbol{\phi})\right) \odot\left(\mathbf{J}_{\zeta} \mathbf{D}(\boldsymbol{\phi})\right)\right] \overrightarrow{\boldsymbol{\sigma}}_{\mathbf{s}}^{2}+\sigma_{n}^{2} \mathbf{1} .
$$

Here $\mathbf{1}=\left[\mathbf{e}_{1}^{T}, \mathbf{e}_{2}^{T}, \ldots \mathbf{e}_{M_{e}}^{T}\right]^{T}$, where $\left\{\mathbf{e}_{p}\right\}_{l=1}^{M_{e}}, l=1,2, \ldots, M_{e}$, are the $M_{e} \times 1$ vectors with one at the $p$-th position and nought otherwise. $\vec{\sigma}_{\mathbf{s}}^{2}$ is a column vector composed of nonzero elements on the diagonal of $\mathbf{R}_{\mathbf{s}}$. The virtual array response matrix $\left(\mathbf{J}_{\zeta}^{*} \mathbf{D}^{*}(\boldsymbol{\phi})\right) \odot\left(\mathbf{J}_{\zeta} \mathbf{D}(\boldsymbol{\phi})\right) \in$ $\mathbb{C}^{M_{e}^{2} \times P}$ can be formulated as

$$
\left(\mathbf{J}_{\zeta}^{*} \mathbf{D}^{*}(\boldsymbol{\phi})\right) \odot\left(\mathbf{J}_{\zeta} \mathbf{D}(\boldsymbol{\phi})\right)=\mathbf{G B}(\boldsymbol{\phi}),
$$


here $\mathbf{B}(\boldsymbol{\phi}) \in \mathbb{C}^{\left(2 M_{e}-1\right) \times P}$ is a dimension-reduced virtual array response matrix that expressed as

$$
\mathbf{B}(\boldsymbol{\phi})=\left[\mathbf{b}\left(\phi_{1}\right), \mathbf{b}\left(\phi_{2}\right), \ldots, \mathbf{b}\left(\phi_{p}\right), \ldots \mathbf{b}\left(\phi_{P}\right)\right],
$$

where

$$
\mathbf{b}\left(\phi_{p}\right)=\left[e^{-j\left(M_{e}-1\right) \phi_{p}}, \ldots, e^{-j \phi_{p}}, 1, e^{j \phi_{p}}, \ldots e^{j\left(M_{e}-1\right) \phi_{p}}\right]^{T},
$$

and $\mathbf{G} \in \mathbb{C}^{M_{e}^{2} \times\left(2 M_{e}-1\right)}$ is given by

$$
\mathbf{G}=\left(\mathbf{J}_{\zeta} \otimes \mathbf{J}_{\zeta}\right) \mathbf{H},
$$

where $\otimes$ symbolises Kronecker product. Here $\mathbf{H}$ is the selection matrix [9] of ULA given by

$$
\mathbf{H}=\left[\operatorname{vec}\left(\mathbf{H}_{M_{e}-1}\right), \cdots, \operatorname{vec}\left(\mathbf{H}_{1}\right), \operatorname{vec}\left(\mathbf{H}_{0}\right), \operatorname{vec}\left(\mathbf{H}_{1}^{T}\right), \cdots, \operatorname{vec}\left(\mathbf{H}_{M_{e}-1}^{T}\right)\right]
$$

with

$$
\mathbf{H}_{i}=\left[\begin{array}{cc}
\mathbf{0}_{M_{e}-i} & \mathbf{I}_{M_{e}-i} \\
\mathbf{0}_{i, i} & \mathbf{0}_{i, M_{e}-i}
\end{array}\right], i=0,1, \ldots M_{e}-1 .
$$

As is known from (20), the selection matrix $\mathbf{G}$ of the virtual ULA is just a derivation of $\mathbf{H}$, that is, an inner product of $\mathbf{J}_{\zeta} \otimes \mathbf{J}_{\zeta}$ and the selection matrix of ULA. (16) can be reformulated as below,

$$
\mathbf{Y}=M \mathbf{G B}(\boldsymbol{\phi}) \overrightarrow{\boldsymbol{\sigma}}_{\mathrm{s}}^{2}+\sigma_{n}^{2} \mathbf{1} .
$$

From (23), we know that the observation data $\mathbf{Y}$, vectorization of $\mathbf{R}_{\mathbf{y}}$, behaves like a new signal model. $\overrightarrow{\boldsymbol{\sigma}}_{\mathrm{s}}^{2}$ is the equivalent signal vector, which describes the power of each signal. The virtual array response matrix $\mathbf{G B}(\boldsymbol{\phi})$ is a new observation matrix, which has a larger aperture than the array which is not vectorized. $\sigma_{n}^{2}$ represents the power of noise. When no knowledge of noise is available, $\sigma_{n}^{2}$ is estimated and given by the minimum of the eigenvalue of $\mathbf{R}_{\mathbf{y}}$. From [28], we know that $\operatorname{Rank}(\mathbf{G B}(\boldsymbol{\phi}))$ is $P$, which satisfies $P<2 H_{e}+1$. So if any complete basis of $P$-dimension vectors is given, (23) can be expressed as a linear combination of the signal powers in the $P$-dimension vectors space.

Remark 1 In [9] and [28], the dimension of the array manifold matrix is reduced from $M^{2} \times P$ to $(2 M-1) \times P$ by Khatri-Rao product. It works for ULA, but not for UCA. For an arbitrary array, the array response matrix is generally expressed as $\left(\mathbf{A}^{*} \odot \mathbf{A}\right) \in \mathbb{C}^{M^{2} \times P}$. But for UCA, having vectorized the observation data by BT technique, the response matrix is reduced to $\mathbf{B} \in \mathbb{C}^{\left(4 H_{e}+1\right) \times P}$. According to the spatial sampling criterion of $H_{e}<M / 2$, we know that if $M>10$, then $M^{2} \simeq(5 \sim 6)\left(4 H_{e}+1\right)$, that is, the array response matrix is reduced from $M^{2} \times P$ to $\left(4 H_{e}+1\right) \times P$.

\subsection{Sparse beamspace DOA estimation via single measurement vector}

Assuming that the overcomplete basis $\left\{\mathbf{b}\left(\widetilde{\phi}_{q}\right)\right\}_{q=1}^{Q}\left(Q \gg M_{e}^{2}\right)$, where $\left\{\widetilde{\phi}_{q}\right\}_{q=1}^{Q}, q=$ $1,2, \cdots, Q$, are the discrete samples from the potential incident directions of signals in beamspace domain. Here denote $\left\{\widetilde{\phi}_{q}\right\}_{q=1}^{Q}$ by the vector $\tilde{\boldsymbol{\phi}}$. Therefore, (23) can be reformulated as the SMV form

$$
\mathbf{Y}=\operatorname{MGB}(\widetilde{\boldsymbol{\phi}}) \mathbf{u}+\sigma_{n}^{2} \mathbf{1}
$$




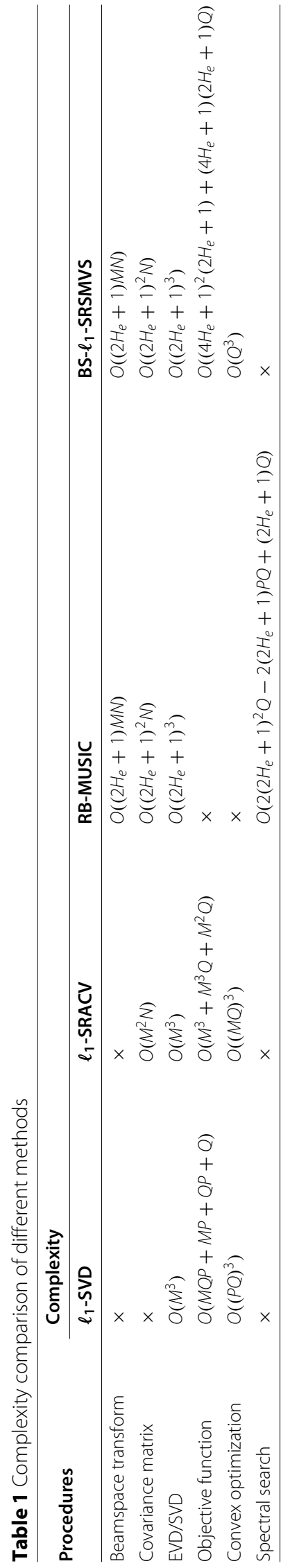




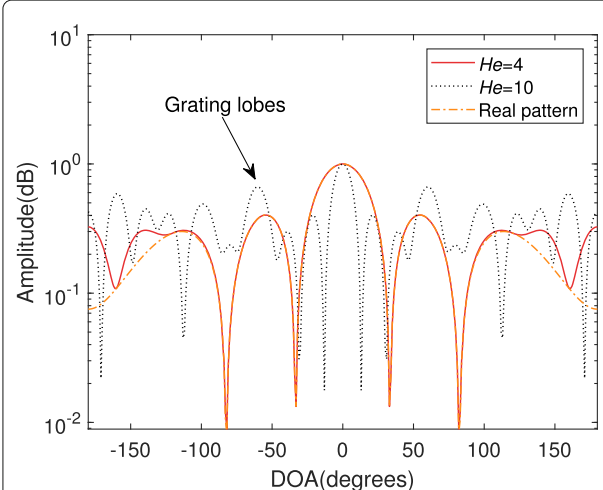

(a)

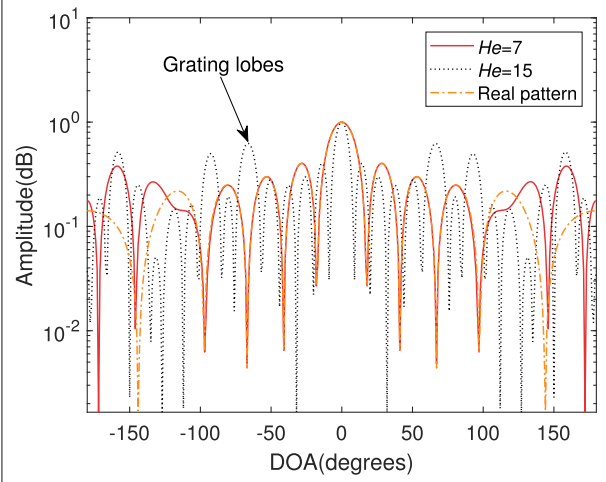

(c)

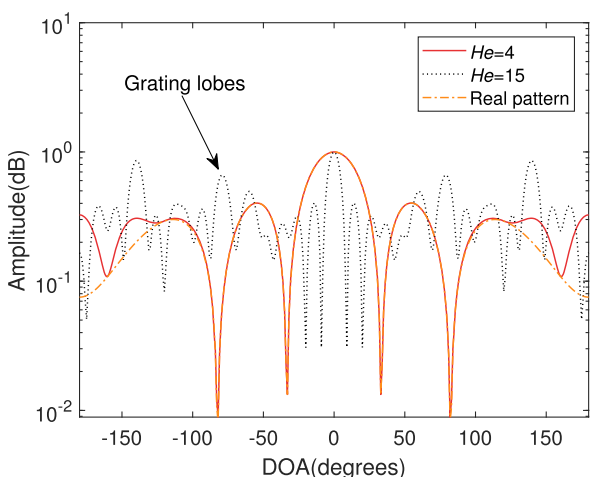

(b)

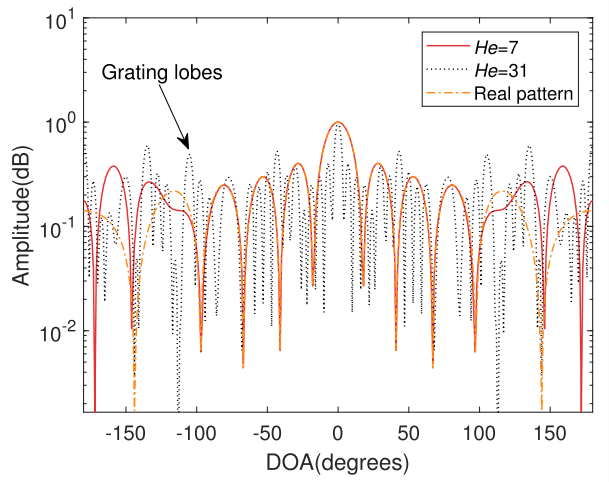

(d)

Fig. 1 Comparison of pattern amplitudes for the $M$-sensor UCA. (a) $M=9, H_{e}$ is 4,10 , correspondingly $r=0.6 \lambda$, 1.5 $\lambda$. (b) $M=9, H_{e}$ is 4,15 , correspondingly $r=0.4 \lambda, 1.5 \lambda$. (c) $M=16, H_{e}$ is 7,15 , correspondingly $r=0.8 \lambda, 0.4 \lambda$. (d) $M=16, H_{e}$ is 7,31 , correspondingly $r=0.8 \lambda, 0.2 \lambda$

which is essentially an underdetermined signal reconstruction problem. We can estimate the DOAs of the signals by recovering the sparse vector $\mathbf{u}$ of the single measurement vector $\mathbf{Y}$. At this point, if the grid resolution of $\widetilde{\boldsymbol{\phi}}$ is dense enough, then some $P$ column vectors of $\mathbf{B}(\widetilde{\boldsymbol{\phi}})$ are approaching to or equal to $\left\{\mathbf{b}\left(\phi_{p}\right)\right\}_{p=1}^{P}$. Correspondingly, a $P$-sparse vector $\tilde{\mathbf{u}}$ is estimated, whose nonzero elements are close to or equal to $\left\{\sigma_{s p}^{2}\right\}_{p=1}^{P}$. In theory, (24) can be solved by the following constraint $\ell_{1}$ optimization that expressed as [29]

$$
\min _{\tilde{\mathbf{u}}}\|\tilde{\mathbf{u}}\|_{1} \text { subject to } \tilde{\mathbf{Y}}=M \mathbf{G B}(\tilde{\boldsymbol{\phi}}) \tilde{\mathbf{u}}+\sigma_{n}^{2} \mathbf{1},
$$

here $\tilde{\mathbf{u}}$ and $\tilde{\mathbf{Y}}$ are the estimates of $\mathbf{u}$ and $\mathbf{Y}$ respectively. From (25), we know that if $\tilde{\mathbf{u}}$ is approaching to $\overrightarrow{\boldsymbol{\sigma}}_{\mathrm{s}}^{2}$, then $\tilde{\mathbf{Y}}$ approximates to $\mathbf{Y}$. And some $\{\widetilde{\phi}\}_{p=1}^{P}$ are very close to the DOAs of the incident signals. We know that the estimate error of $\widetilde{\mathbf{Y}}-\mathbf{Y}$ with the weighted matrix of $\mathbf{W}=\frac{1}{N} \mathbf{R}_{\mathbf{y}}^{T} \otimes \mathbf{R}_{\mathbf{y}}$ follows asymptotically normal (AsN) distribution[29], which is given by

$$
\mathbf{W}^{-\frac{1}{2}}\left[\tilde{\mathbf{Y}}-\operatorname{MGB}(\tilde{\boldsymbol{\phi}}) \tilde{\mathbf{u}}-\sigma_{n}^{2} \mathbf{1}\right] \sim \operatorname{AsN}\left(\mathbf{0}, \mathbf{I}_{M_{e}^{2}}\right) .
$$

Using least-squares criterion, the weighted estimate error follows asymptotic chi-square distribution with $M_{e}^{2}$ degree-of-freedoms. It is formulated as

$$
\left\|\mathbf{W}^{-\frac{1}{2}}\left[\tilde{\mathbf{Y}}-M \mathbf{G B}(\tilde{\boldsymbol{\phi}}) \tilde{\mathbf{u}}-\sigma_{n}^{2} \mathbf{1}\right]\right\|_{2}^{2} \sim A s \chi^{2}\left(M_{e}^{2}\right),
$$


here $\widetilde{\mathbf{Y}}=\operatorname{vec}\left(\widetilde{\mathbf{R}}_{\mathbf{y}}\right)$, where $\widetilde{\mathbf{R}}_{\mathbf{y}}=\frac{1}{N} \sum_{t=1}^{N} \mathbf{y}(t) \mathbf{y}^{H}(t)$. Thus, a modified DOA estimation mode is derived from (25) by introducing the parameter of $\beta$, which makes the inequality $\left\|\mathbf{W}^{-\frac{1}{2}}(\tilde{\mathbf{Y}}-\mathbf{Y})\right\|_{2}^{2} \leqslant \beta^{2}$ hold with a high probability $\tilde{p}$. It specifies how much estimate error we wish to allow. It is expressed as follows

$$
\mathrm{P}\left(\chi^{2}\left(M_{e}^{2}\right) \leqslant \beta^{2}\right)=\tilde{p},
$$

where $\mathrm{P}(\bullet)$ denotes the probability distribution function. For the probability value of $\tilde{p}$, by looking up the probability table of chi-square distribution, we have the regularization parameter $\beta$, that is, $\beta=\sqrt{\chi_{\tilde{p}}^{2}\left(M_{e}^{2}\right)}$. Then, (25) can be expressed as

$$
\min _{\tilde{\mathbf{u}}}\|\tilde{\mathbf{u}}\|_{1} \text { subject to }\left\|\mathbf{W}^{-\frac{1}{2}}\left(\widetilde{\mathbf{Y}}-M \mathbf{G B}(\widetilde{\boldsymbol{\phi}}) \tilde{\mathbf{u}}-\sigma_{n}^{2} \mathbf{1}\right)\right\|_{2}^{2} \leqslant \beta^{2} .
$$

Using the Matlab convex optimization toolbox, the $P$-sparse vector $\tilde{\mathbf{u}}$ can be obtained. We can plot the peaks versus the directions and determine the DOAs of the incoming signals. The proposed method is summarized in Algorithm 1.

Remark 2 From (16), we know that $\operatorname{krank}(\mathbf{G B}(\boldsymbol{\phi})) \geq \min \left\{P, 2 \times \operatorname{krank}\left(\boldsymbol{J}_{\zeta} \boldsymbol{D}(\boldsymbol{\phi})\right)-1\right\}$. Here $\operatorname{krank}\left(\mathbf{J}_{\zeta} \boldsymbol{D}(\boldsymbol{\phi})\right)=M_{e}$, where $\operatorname{krank}(\bullet)$ denotes the Kruskal rank (see definition in [28] for details). It means that every collection of $2 M_{e}-1$ column vectors of $\mathbf{G B}(\boldsymbol{\phi})$ is linearly independent and there exits a set of $2 M_{e}$ column vectors linearly dependent. That is $\operatorname{Spark}(\mathbf{G B}(\boldsymbol{\phi}))=2 M_{e}$. The constraint condition of $\ell_{1}$ optimization for a unique P-sparse vector $\mathbf{u}$ is $\operatorname{Spark}(\mathbf{G B}(\boldsymbol{\phi}))>2 P$. i.e. $2 H_{e}+1>P$, which means that the DOA estimator for virtual ULA with $H_{e}$ modes can handle $2 H_{e}$ signals at most.

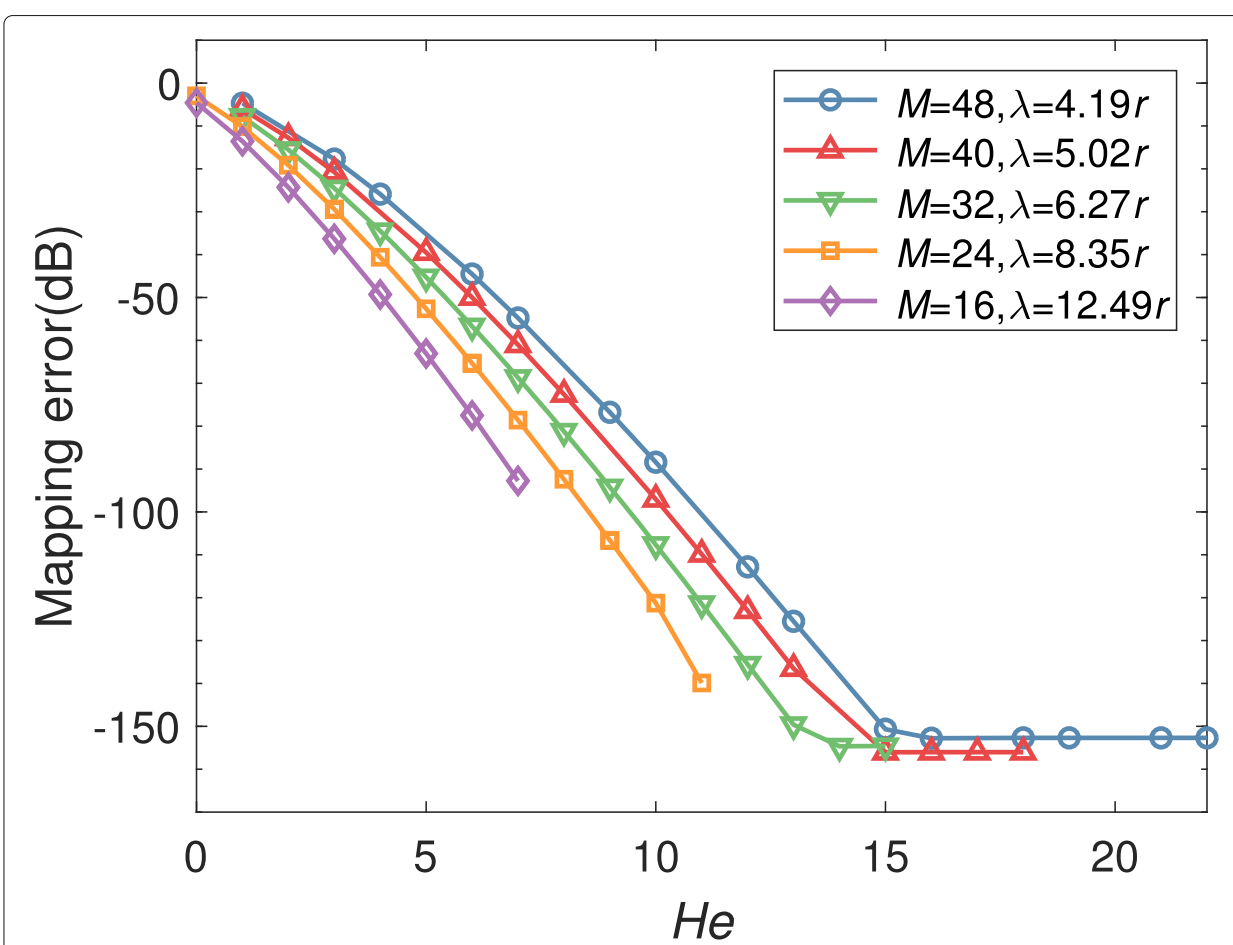

Fig. 2 Mapping error as a function of phase mode $H_{e}$ for UCA with $r / \lambda$, in which the circumferential spacing between adjacent sensors is $0.0313 r$. The maximum of phase mode is respectively $7,11,15,18$, and 22 , correspondingly $M=16,24,32,40,48$ 


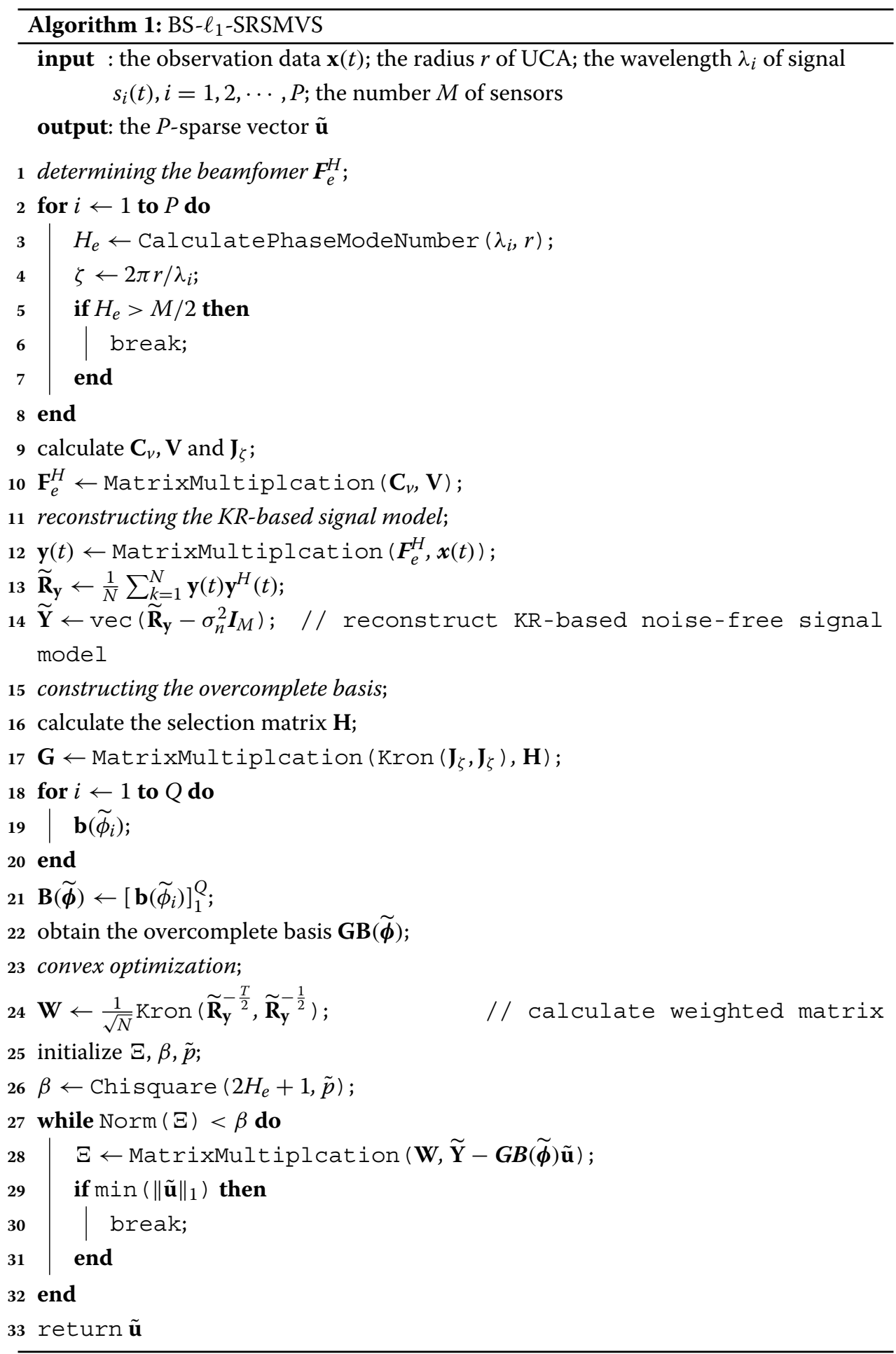

\section{Computational complexity analysis}

The methods of $\ell_{1}$-SVD and $\ell_{1}$-SRACV have advantages of being applicable for an arbitrary array; however, they have much higher complexity than the proposed method. Except for the optimization calculation of objective function, for $\ell_{1}$-SVD, the computational load is mainly stressed on singular value decomposition of observation data, and 
for $\ell_{1}$-SRACV, the computational cost is concerned on eigenvalue decomposition (EVD) of covariance matrix. The proposed method reduces the computational complexity and lowers the dimensionality of observation data using BT technique. Table 1 shows the computational complexity of some different methods such as $\ell_{1}$-SVD, $\ell_{1}$-SRACV, BS- $\ell_{1}$ SRSMVS, and RB-MUSIC. We know that a priori number of incoming signals should be necessary for $\ell_{1}$-SVD and RB-MUSIC. So their computational costs have a close relationship with $P \cdot \ell_{1}$-SRACV is not susceptible to the number $P$ of signals but the number $M$ of sensors. Here $M>P$ and $M>2 H_{e}$. From Table 1, we know that the computational complexity of the proposed method is $O\left(\left(2 H_{e}+1\right) M N+\left(2 H_{e}+1\right)^{2} N+\left(2 H_{e}+1\right)^{3}+\right.$ $\left.\left(4 H_{e}+2\right)\left(4 H_{e}+1\right)\left(2 H_{e}+1\right) Q+Q^{3}\right)$, which covers the process of beamspace transformation, covariance matrix, eigenvalue decomposition (skipping or skimming if the noise power is known in advance), the construction of objective function via weighted matrix $\mathbf{W}^{-\frac{1}{2}}$ and optimization calculation without spectral search. Considering the estimation accuracy of DOA, $Q$ should be far greater than $M$; thus, it is the most-weighted factor that results in major time consumption in the estimators. Provided that the grid resolution of directional samples $Q$ is constant, we know that the larger the number of $M$ or $P$ is, the higher the computational complexity is. In addition, due to the dimension-reduction process of UCA, the computational complexity of covariance matrix and EVD of the proposed method is lower than that of $\ell_{1}$-SVD and $\ell_{1}$-SRACV. Thus, its computational load is much less than $\ell_{1}$-SVD and $\ell_{1}$-SRACV. Comparing the subspace-based method as RB-MUSIC, our proposed method has no advantages of computational complexity, but the superiority of angular separation that conventional subspace-based methods are incomparable, which has been illustrated in Section 5 .

\section{Results and discussion}

In this section, we evaluate the performance of the proposed method by simulations with different settings. We run some independent trials on a $\mathrm{PC}$ with a $2.4-\mathrm{GHz}$ processor of Intel Core i5-6200U, 8G of RAM. The software environment is Matlab 2017b running on Windows 10 operating system.

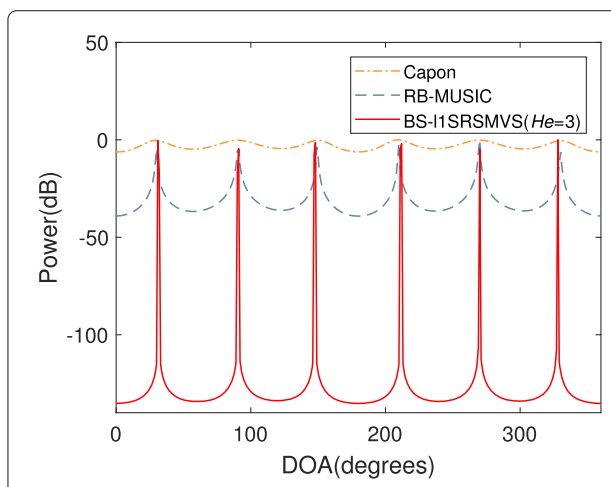

(a)

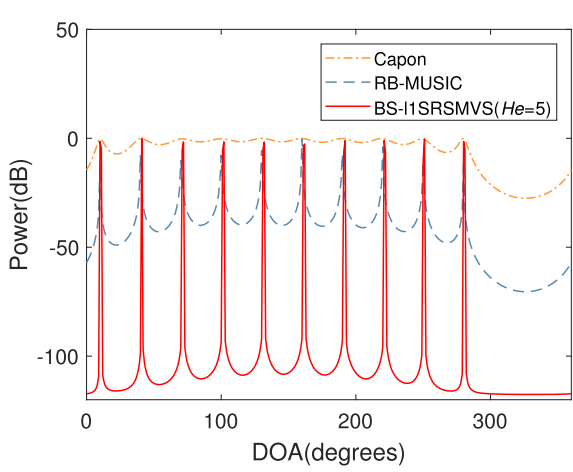

(b)

Fig. 3 Spatial spectra for Capon beamformer, RB-MUSIC and BS- $\ell_{1}$-SRSMVS. a $M=7, r / \lambda=0.5, N=1000$, SNR=0, $H_{e}=3, \mathrm{DOAs}=\left(30^{\circ}, 90^{\circ}, 150^{\circ}, 210^{\circ}, 270^{\circ}, 330^{\circ}\right) . \mathbf{b} M=11, r / \lambda=0.5, N=2000, S N R=20, H_{e}=5, \mathrm{DOAs}=\left(10^{\circ}, 40^{\circ}\right.$, $\left.70^{\circ}, 100^{\circ}, 130^{\circ}, 160^{\circ}, 190^{\circ}, 220^{\circ}, 250^{\circ}, 280^{\circ}\right)$ 


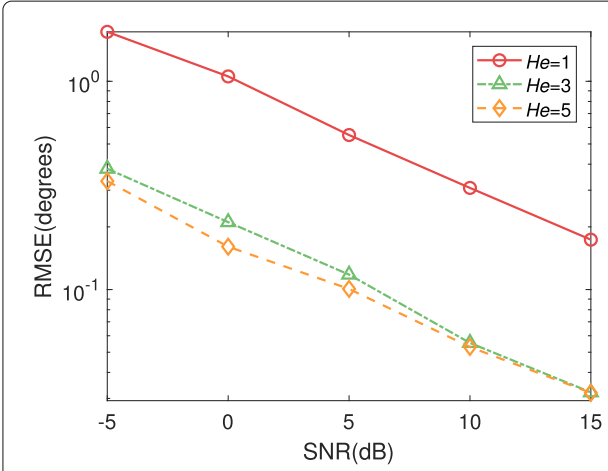

(a)

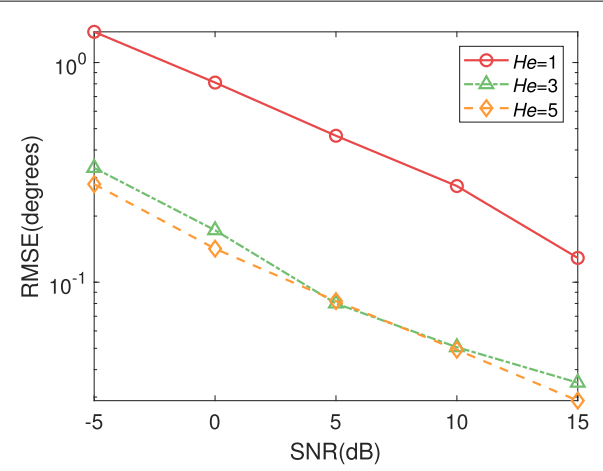

(b)

Fig. 4 RMSEs versus SNR with different phase modes. a $M=24$. b $M=32$

\subsection{Phase mode choice and mapping error}

In this subsection, we run some experiments to demonstrate the relationship between the amplitude of steering vectors and phase modes. In addition, the mapping error of steering vectors on transforming UCA to virtual ULA is defined, and its relationship with phase mode is given as well. Here taking a 9-sensor UCA and a 16-sensor UCA as examples, the amplitude of steering vectors of different phase modes is depicted in Fig. 1. For the 9-sensor UCA with the maximal phase mode $H_{e}$ being 4 , we can see from the Fig. 1(a) that the maximal amplitude of grating lobes is $4 \mathrm{~dB}$ lower than that of $H_{e}=10$. Similarly, the maximum of grating lobes is $3 \mathrm{~dB}$ lower than that of $H_{e}=15$, which is plotted in Fig. 1(b). For the 16-sensor UCA with the maximal phase mode $H_{e}$ being 7 , it is noticed that the maximal amplitude of grating lobes of $H_{e}=15$ ( $\lambda$ is $0.8 r$ correspondingly) in Fig. 1(c) is almost $5 \mathrm{~dB}$ higher than that of $H_{e}=7$ and the maximum of grating lobes of $H_{e}=31$ ( $\lambda$ is $0.4 r$ correspondingly) in Fig. $1(\mathrm{~d})$ is about $7 \mathrm{~dB}$ higher than that of $H_{e}=7$. It is noteworthy that with the phase mode increasing, the main lobe of $H_{e}=15, H_{e}=31$ is almost $10^{\circ}$ and $14^{\circ}$ narrower than that of $H_{e}=7$ for the 16-sensor UCA, and the main lobe of $H_{e}=10, H_{e}=15$ is about $18^{\circ}$ and $22^{\circ}$ narrower than that of $H_{e}=4$ for the 9sensor UCA. Taking the phase mode of $H_{e}=7$ for the 16-sensor UCA with radius $r=1$

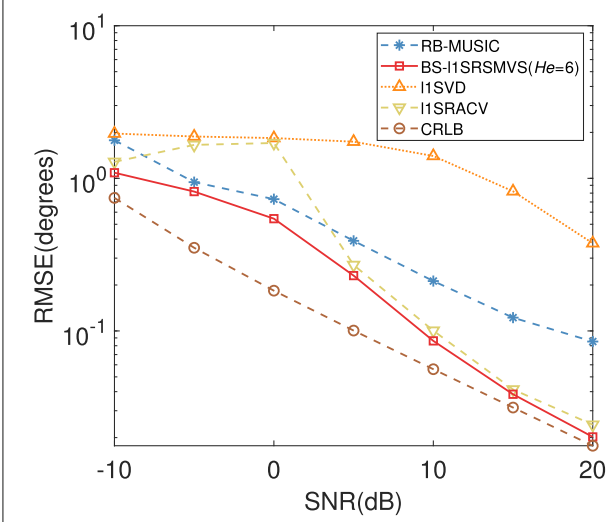

(a)

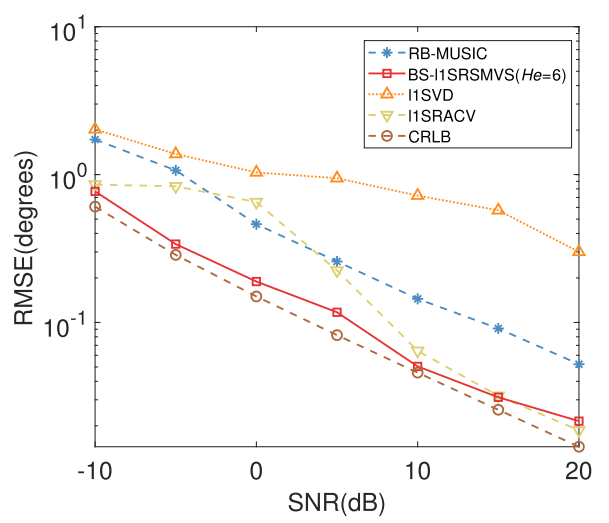

(b)

Fig. 5 RMSEs versus SNR with RB-MUSIC, BS- $\ell_{1}-S R S M V S, \ell_{1}-S V D, \ell_{1}-S R A C V$ and CRLB. a $M=13, N=500$. b $M=13, N=1000$ 
for example, the circumferential spacing between adjacent array sensors is 0.39 , which is larger than half the wavelength when $H_{e}=15$. It means that the phase ambiguity of steering vectors may be occurred in the process of UCA's transforming to virtual ULA if the maximum of phase mode does not satisfy $H_{e}<M / 2$.

Using (8), we can get the steering vectors with Vandermonde structure of a new ULAtype array. Under the constraints of the number of sensors and interelement spacing, the steering vectors of this new array are the finite approximation of the far-field pattern with phase mode excitation. It is noted that this constraint of $M>2 H_{e}$ is similar to the Nyquist sampling criterion, in which $H_{e}$ defines the maximum spatial frequency component in the array excitation [27]. When $H_{e}=\zeta$, it is obvious that the circumferential spacing between adjacent sensors of UCA is less than $0.5 \lambda$, which sufficiently avoids spatial aliasing in the virtual ULA. The approximation leads to mapping error. Here we define it as

$$
\epsilon_{h}\left(H_{e}\right)=\frac{\left\|\mathbf{a}\left(\phi_{p}\right)-\sqrt{M} \mathbf{F}_{e} \mathbf{J}_{\zeta} \mathbf{d}\left(\phi_{p}\right)\right\|_{\mathrm{F}}}{\left\|\mathbf{a}\left(\phi_{p}\right)\right\|_{\mathrm{F}}}
$$

Suppose $H_{e}=\lfloor n \zeta\rfloor, n \in \mathbb{Q}^{+}$, which $\mathbb{Q}^{+}$is the positive rational number set. Figure 2 shows that mapping error $\epsilon_{h}\left(H_{e}\right)$ decreases as $H_{e}$ (or $\left.n\right)$ increases and $r / \lambda$ decreases. The plots show the mapping error as a function of phase mode $H_{e}$ of the UCA with $M$ being respectively 48, 40, 32, 24, 16 and $\lambda$ being correspondingly $4.19 r, 5.02 r, 6.27 r, 8.35 r, 12.49 r$. Their circumferential spacings between adjacent sensors of the UCAs are all less than $0.5 \lambda$. From Fig. 2, we know that $\epsilon_{h}\left(H_{e}\right) \approx 0$ (i.e. the minimum is approaching to $10^{-15}$ ) if $H_{e}$ approximates to $\infty$. Accordingly, the interelement spacing is approaching to 0 theoretically, and at this point, (8) represents the far-field pattern of the continuous circular aperture. So if extending $H_{e}$ large enough, the mapping error is almost negligible.

\subsection{Spectra of the proposed method}

We consider the cases of two UCAs, the sensors' number of which is respectively $M=7$ and $M=11$, and we have the performance comparisons between BS- $\ell_{1}$-SRSMVS, Capon beamformer and RB-MUSIC.

Figure 3 shows the spatial spectra of BS- $\ell_{1}$-SRSMVS, Capon beamformer and RBMUSIC. One case is investigated, where the true DOAs of actual narrowband signals

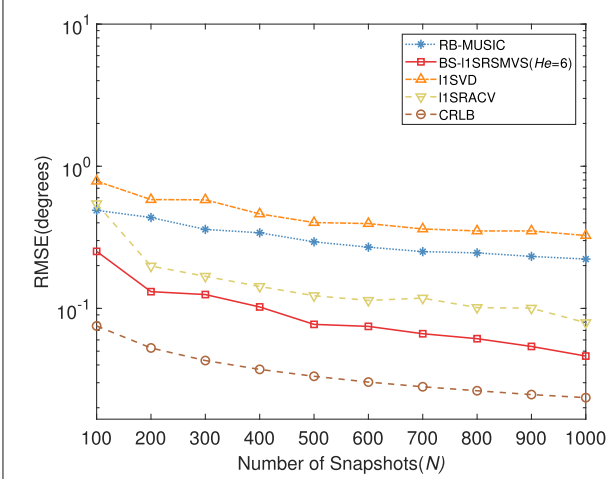

(a)

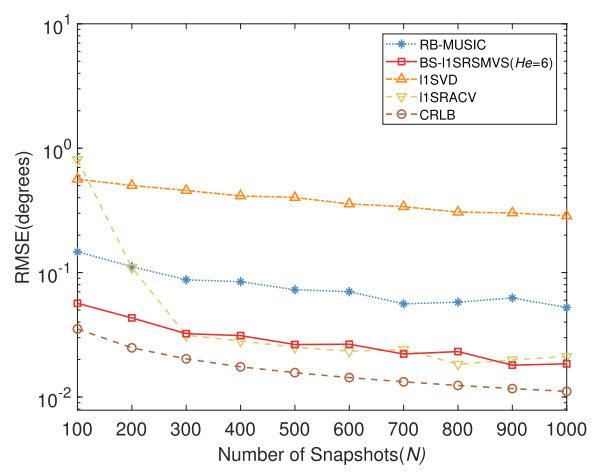

(b)

Fig. 6 RMSEs versus snapshots with RB-MUSIC, BS- $\ell_{1}-$ SRSMVS, $\ell_{1}-S V D, \ell_{1}-S R A C V$ and CRLB. a $M=13$, $S N R=0 d B . \mathbf{b} M=13, S N R=10 \mathrm{~dB}$ 
impinging on the array are respectively $30^{\circ}, 90^{\circ}, 150^{\circ}, 210^{\circ}, 270^{\circ}$, and $330^{\circ}$. And the simulation results are plotted in Fig. 3(a), in which the number of sensors is $M=7, r / \lambda=$ $0.5, \mathrm{SNR}=0 \mathrm{~dB}$ and the number of snapshots is $N=1000$, the parameter of $\beta$ is 7.07 . The other case which $M=11, r / \lambda=0.5, \mathrm{SNR}=20 \mathrm{~dB}$ and $N=2000$ is considered. The signals impinge on the array from the directions of $10^{\circ}, 40^{\circ}, 70^{\circ}, 100^{\circ}, 130^{\circ}, 160^{\circ}, 190^{\circ}, 220^{\circ}$, $250^{\circ}$, and $280^{\circ}$. The simulation results are depicted in Fig. 3(b), in which the parameter of $\beta$ is 7.28. As is known that the maximum phase mode of the 7-sensor UCA is 3 and that of 11 -sensor UCA is 5 . From the plots, we know that RB-MUSIC and BS- $\ell_{1}$-SRSMVS are both capable of estimating all these signals; however, BS- $\ell_{1}$-SRSMVS has higher resolution and output SNR than those of RB-MUSIC and Capon beamformer. We also notice that the maxima of DOAs estimated of these two cases are 6 and 10. It is indicated that BS$\ell_{1}$-SRSMVS can estimate $2 H_{e}$ signals regardless of the number of sensors on the premise of $M>2 H_{e}$. Thus for an $M$-sensor UCA, the proposed method can estimate at most $M-1$ signals.

\subsection{Performance evaluation}

In this subsection, we carry out independent trials to verify the performance of our proposed method provided that the phase mode of $H_{e}$ is $1,3,5$ respectively. The number of sensors is 24,32. The radius $r$ of the UCA is $0.5 \lambda$. The root mean squared error is defined as RMSE $=\sqrt{\mathrm{E}\left[\frac{1}{Q} \sum_{j=1}^{Q}\left(\tilde{\theta}_{j}-\theta_{j}\right)^{2}\right]}$, where $\tilde{\theta}_{j}$ indicates the estimated DOA and $\theta_{j}$ denotes the true DOA. $Q$ is the number of independent Monte Carlo experiments with $Q=500$. SNR ranges from -5 to $15 \mathrm{~dB}$. We know that on the transforming of UCA in elementspace domain to virtual ULA in beamspace domain, the mapping error decreases as $H_{e}$ increases. Likewise, as is seen from the RMSEs shown in Fig. 4, we know that the RMSEs decrease as the phase modes increase, and with SNR increasing, RMSEs decrease as well.

We compare the RMSEs of the proposed method, other methods and the stochastic Cramér-Rao lower bound (CRLB) [30], in which the number of sensors is 13 and the radius of the UCA is $0.5 \lambda$. Figure 5 shows the RMSEs of different methods under different SNR conditions. The statistical results are obtained by running the Monte Carlo simulation of 500 independent trials, where the number of snapshots is respectively 500, 1000 and SNR ranges from -10 to $20 \mathrm{~dB}$ in $5 \mathrm{~dB}$ steps. The plots are shown in Fig. 5(a) and (b). With SNR increasing, it can be seen that the RMSEs of the proposed method are statistically less than that of $\ell_{1}$-SVD, $\ell_{1}$-SRACV, and RB-MUSIC.

The RMSEs varying with the number of snapshots are plotted in Fig. 6, where SNR is respectively $0 \mathrm{~dB}$ and $10 \mathrm{~dB}$ and the number of snapshots ranges from 100 to 1000 . From the figure, we can see that with the increase of the snapshots, the RMSEs of these methods are all decrease no matter what SNR is. However, the proposed method and $\ell_{1}$ SRACV both have lower RMSE than other methods. Meanwhile, the proposed method yields much smaller RMSE than $\ell_{1}$-SRACV when the number of snapshots is less than 300. Our proposed method outperforms these SSR-based DOA estimation methods as $\ell_{1}$-SVD and $\ell_{1}$-SRACV as a whole.

\subsection{Performance of angular separation}

We know that near the direction where the array manifold vector changes rapidly, the corresponding angular resolution is higher as well with the change of the DOAs. Thus, 
we define the directional derivative of the steering vector and apply its Euclidean norm to characterize the angular resolution. Generally for UCA, the coefficient $\rho\left(\phi_{p}\right)$ that reflects the angular separation is expressed as

$$
\begin{aligned}
\rho\left(\phi_{p}\right) & =\left\|\frac{d \mathbf{a}\left(\phi_{p}\right)}{d \phi_{p}}\right\|_{2}, \quad \forall p=1, \cdots, P \\
& =\zeta \sqrt{\sum_{i=1}^{M} \sin ^{2}\left(\phi_{p}-\gamma_{i}\right), \quad i=1, \cdots, M .}
\end{aligned}
$$

The larger $\rho\left(\phi_{p}\right)$ is, the higher the performance is. From (31), we know that it is related to the number of sensors and the sensors' position around UCA. In theory, if $M=2$, we can obtain $\rho\left(\phi_{p}\right)=\zeta \sqrt{1-\cos \left(\frac{2 \pi}{M}\right) \cos \left(2 \phi_{p}-\frac{2 \pi}{M}\right)}$ and the maximal separation angle is $\pi / M$. Different from the subspace-based DOA methods, the SSR-based DOA estimation methods are just dependent on the grid resolution of direction samples [8]. Theoretically, if enough SNR, the maximal separation angle is much smaller than that of the subspacebased DOA methods.

In this subsection, we compare the performance of angular separation of the proposed method with that of MUSIC, RB-MUSIC, $\ell_{1}$-SVD and $\ell_{1}$-SRACV. In these compared methods, the first two are applicable in element-space domain and beamspace domain based on subspace decomposition, the last two are SSR-based methods in element-space domain. The simulation is based on a 13-sensor UCA with half-wavelength circumferential element spacing. The number of snapshots $N$ is 200 and 2000 respectively. The grid resolution of the overcomplete basis is $0.1^{\circ}$ with 1800 points sampled from $0^{\circ}$ to $180^{\circ}$.

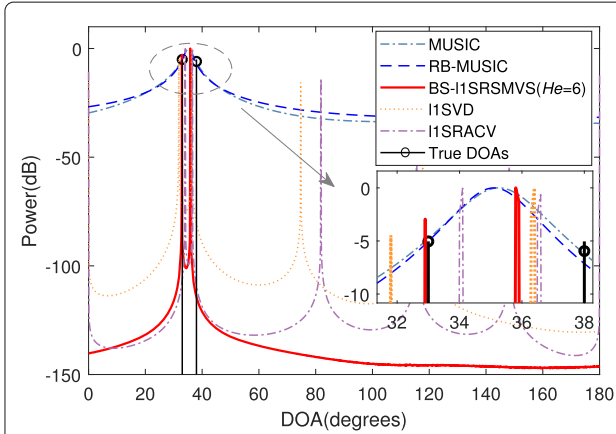

(a)

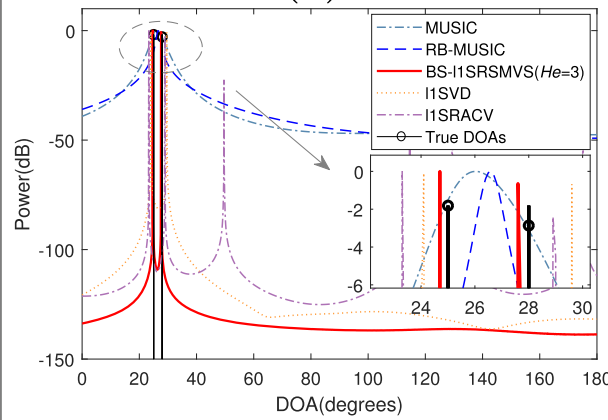

(c)

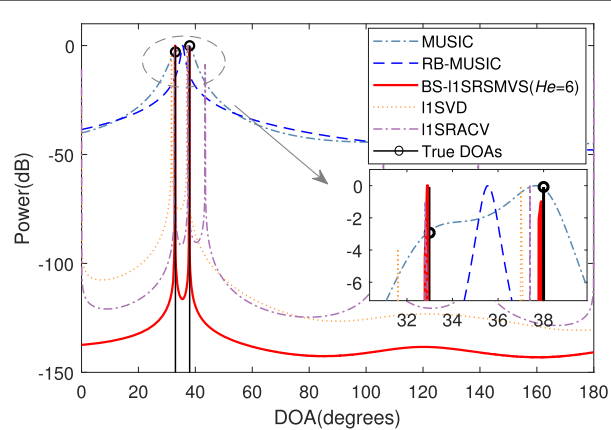

(b)

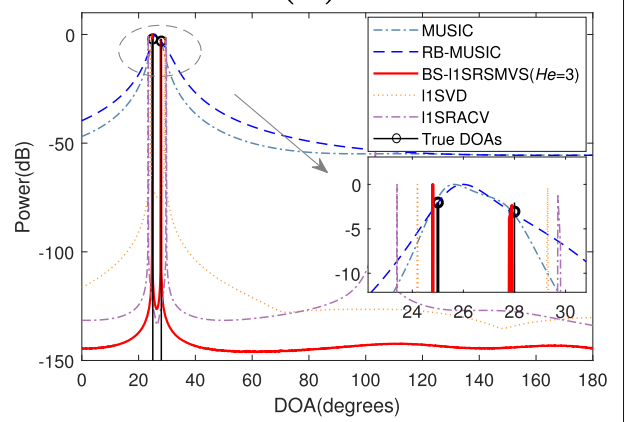

(d)

Fig. 7 Angular separation versus $S N R$ of different methods, $M=13, r / \lambda=0.5$. a $S N R=0 d B, N=2000$, $\mathrm{DOAs}=\left(33^{\circ}, 38^{\circ}\right) \cdot \mathbf{b} \mathrm{SNR}=10 \mathrm{~dB}, \mathrm{~N}=2000, \mathrm{DOAs}=\left(33^{\circ}, 38^{\circ}\right) . \mathbf{c} \mathrm{SNR}=20 \mathrm{~dB}, N=200, \mathrm{DOAs}=\left(25^{\circ}, 28^{\circ}\right) . \mathbf{d}$ $S N R=30 \mathrm{~dB}, N=200, \mathrm{DOAs}=\left(25^{\circ}, 28^{\circ}\right)$ 
The maximal separation angle is $13.85^{\circ}$. Here the angle we have taken is not beyond a half of the value, i.e. $6.9^{\circ}$.

Figure 7 shows the angular resolution versus SNR using different methods. The two sources are spaced closely, they spaced $5^{\circ}$ in Fig. 7(a) and (b), and they are close to $3^{\circ}$ in Fig. 7(c) and (d). From the results, we know that the subspace-based MUSIC merges the two peaks, whereas our proposed method, $\ell_{1}$-SVD and $\ell_{1}$-SRACV are capable of resolving the two sources. From Fig. 7(a) and (b), we know that even if the decrease of SNR, $\ell_{1}$-SVD and $\ell_{1}$-SRACV still have the excellent performance of angular separation, but if SNR decreases to $0 \mathrm{~dB}, \ell_{1}$-SVD produces spurious peaks [6]. And $\ell_{1}$-SRACV produces spurious peaks as well if improper regularization parameters. However, the proposed method still has the perfect separation capability and ideal DOA estimation accuracy even if the number of the signals is unknown.

\subsection{Comparison of computational complexity}

In this subsection, we compare the CPU time of $\ell_{1}$-SVD, $\ell_{1}$-SRACV and that of our proposed method by plotting the CPU time versus the number of snapshots, array sensors and sources. The results are shown in Fig. 8. As is seen from the plots, we know that the SSR-based methods have higher computational complexity than the subspace-based methods as RB-MUSIC, which is verified in Table 1. But comparing other SSR-based methods, the proposed method has greater superiority.

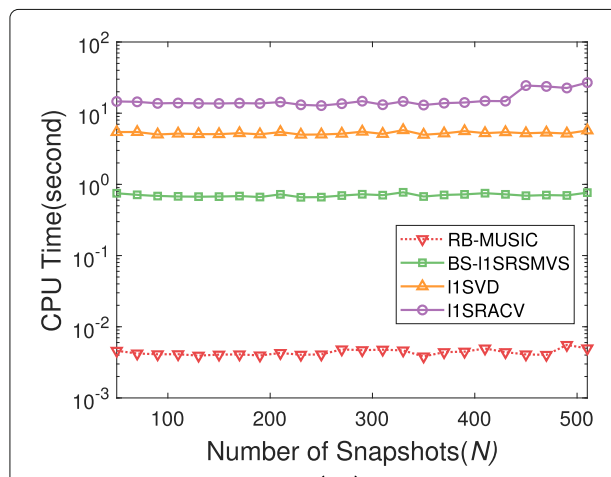

(a)

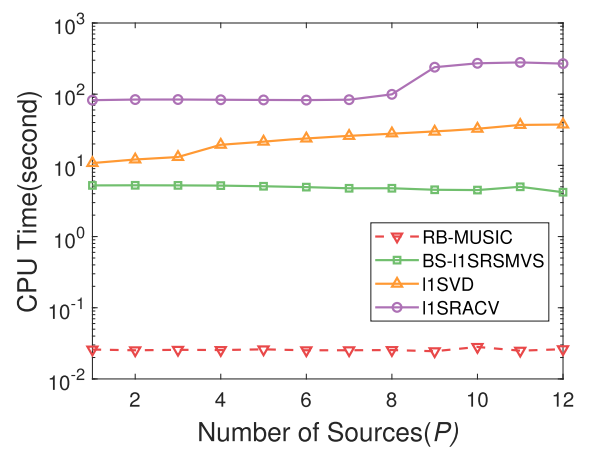

(b)

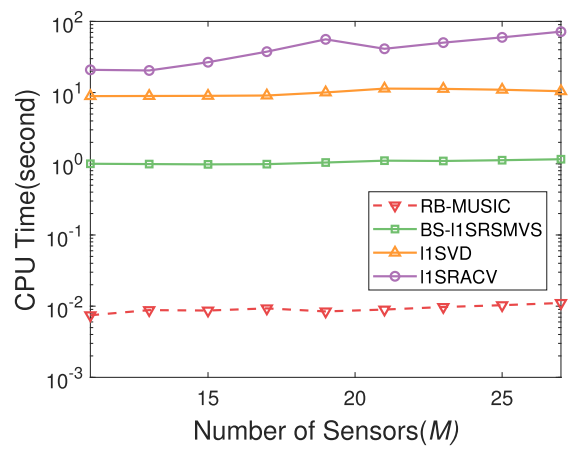

(c)

Fig. 8 CPU time comparisons of BS- $\ell_{1}-S R S M V S, \ell_{1}-S V D, \ell_{1}-S R A C V$ and RB-MUSIC. a CPU time versus snapshots $N, M=18, r / \lambda=0.5$, SNR $=20 \mathrm{~d} B$. $\mathbf{b}$ CPU time versus number of sources $P, M=28, r / \lambda=0.5$, $S N R=20 d B . \mathbf{c} C P U$ time versus number of sensors $M_{1} H_{e}=5, N=500, r / \lambda=0.5, S N R=20 d B$ 
Figure 8(a) shows CPU time versus snapshots using different methods. Here assume $\mathrm{SNR}=20 d B, M=18, \mathrm{DOA}=66^{\circ}, \mathrm{Q}=180$, the number $N$ of snapshots ranges from 50 to 510 . The DOA estimate is measured from 100 Monte Carlo runs. As is seen from the plot, the proposed method has far less time cost than $\ell_{1}$-SVD and $\ell_{1}$-SRACV.

In Fig. 8(b), we plot the CPU time of different methods with the number of sources $P$ increasing, where SNR is $20 \mathrm{~dB}$, the number $M$ of sensors is 28 , the snapshots are assumed 100 and $Q$ is 360 . The DOAs of sources ranges from $10^{\circ}$ to $340^{\circ}$ with $30^{\circ}$ angle spacing between two adjacent sources. As we known from Section 4, the computational complexity of the proposed method has nothing to do with source numbers but the overcomplete basis. Thus, we can see that even if the number of the source increases, the CPU time of the proposed method has never increased dramatically all the time. The CPU time of $\ell_{1}$ SVD is mainly dependent on the number of sources. If the number of sources increases from 1 to 12, accordingly the CPU time ranges from 10.77 seconds to 37.46 seconds.

Figure 8(c) shows CPU time versus the number of sensors using different methods, where SNR is $20 \mathrm{~dB}, M=11,13, \cdots, 27$, the snapshots are 500 . The DOA of one incoming signal is assumed $55^{\circ}$. Comparing the CPU time of $\ell_{1}-\mathrm{SVD}$ and $\ell_{1}$-SRACV, the proposed method has obvious advantages of lower computational complexity. In the case that phase mode $H_{e}$ of the proposed method is fixed at the same value no matter what the number of sensors is, the steering vectors are dimension-reduced to $2 H_{e}+1$. Thus, we know that the CPU time of our proposed method is not relevant to the number of $M$ but with phase mode $H_{e}$, while that of $\ell_{1}$-SRACV does increase with $M$. From the simulation, we know that the computational cost of the proposed method is nearly a tenth that of $\ell_{1}$-SVD and $\ell_{1}$-SRACV.

\section{Conclusion}

In this paper, a low complexity sparse beamspace DOA estimation method for UCA is presented. In the proposed method, the virtual ULA-like array signal model is obtained by using beamspace transform, and having applied the vectorization operation on the covariance matrix of this new signal model, a dimension-reduction signal model is formulated, which greatly reduces the computational complexity. The results show that it not only has better DOA resolution performance than the subspace-based methods, but also has low computational complexity comparing other sparse-like DOA estimation methods in the case of multiple incoherent incoming signals. It should be mentioned that as an SSR-based beamspace DOA estimation method, the DOA estimation accuracy is susceptible to the grid resolution of directions samples of the overcomplete basis, and its virtual beamspace array aperture is restricted by the spatial sampling theorem as well.

\section{Abbreviations}

DOA: Direction-of-arrival; UCA: Uniform circular array; SMV: Single measurement vector; SSR: sparse-signal-representation; BT: Beamspace transform; ULA: Uniform linear array; SNR: Signal-to-noise ratio; MUSIC: Multiple signal classification; RB-MUSIC: Real Beamspace MUSIC; KR: Khatri-Rao; MMV: Multiple measurement vector; SVD: Singular value decomposition; EVD: Eigenvalue decomposition

\section{Acknowledgements}

The authors thank the anonymous reviewers for their enlightening comments and careful reviews, which helped improve the manuscript.

Authors' contributions

All authors contributed to the conception and design of the experiments and the interpretation of simulation results. ZD wrote the software, performed the experiments and data analysis, and wrote the first draft of the manuscript. TW 
substantially revised the manuscript. DZ and LG give some helpful experimental instructions and guidance. All authors read and approved the final manuscript.

\title{
Funding
}

This work was supported in part by The National Key Research and Development Program of China under Grant 2016 YFB0502001.

\section{Declarations}

\section{Competing interests}

The authors declare that they have no competing interests.

\begin{abstract}
Author details
${ }^{1}$ Wireless Network Positioning and Communication Integration Research Center, School of Electronic Engineering, Beijing University of Posts and Telecommunications, 100876 Beijing, China. ${ }^{2}$ The 54th Research Institute of CETC, 050081 Shijiazhuang, China. ${ }^{3}$ State Key Laboratory of Public Big Data, Guizhou Big Data Academy, Guizhou University, 550025 Guiyang, China.
\end{abstract}

Received: 6 March 2021 Accepted: 12 July 2021

Published online: 30 July 2021

\section{References}

1. L. C. Godara, Application of antenna arrays to mobile communications. ii. beam-forming and direction-of-arrival considerations. Proc. IEEE. 85(8), 1195-1245 (1997)

2. Z. Zheng, W. Wang, H. Meng, H. C. So, H. Zhang, Efficient beamspace-based algorithm for two-dimensional doa estimation of incoherently distributed sources in massive mimo systems. IEEE Trans. Veh. Technol. 67(12), 11776-11789 (2018)

3. B. Xu, Y. Zhao, Transmit beamspace-based dod and doa estimation method for bistatic mimo radar. Sig. Process. 157, 88-96 (2019)

4. M. Esfandiari, S. A. Vorobyov, S. Alibani, M. Karimi, Non-iterative subspace-based doa estimation in the presence of nonuniform noise. IEEE Sig. Process. Lett. 26(6), 848-852 (2019)

5. L. Yang, Y. Yang, Y. Zhang, Subspace-based direction of arrival estimation in colored ambient noise environments. Dig. Sig. Process. 99, 102650 (2020). https://doi.org/10.1016/j.dsp.2019.102650

6. Y.Xia, S. Kanna, D. P. Mandic, Maximum likelihood parameter estimation of unbalanced three-phase power signals. IEEE Trans. Instrum. Meas. 67(3), 569-581 (2018)

7. C. F. Mecklenbrauker, P. Gerstoft, in 201927 th European Signal Processing Conference (EUSIPCO), Maximum-likelihood doa estimation at low snr in laplace-like noise (IEEE, Piscataway, 2019), pp. 1-5

8. J. Yin, T. Chen, Direction-of-arrival estimation using a sparse representation of array covariance vectors. IEEE Trans. Sig. Process. 59(9), 4489-4493 (2011)

9. Z. Q. He, Q. H. Liu, L. N. Jin, S. Ouyang, Low complexity method for doa estimation using array covariance matrix sparse representation. Electron. Lett. 49(3), 228-230 (2013)

10. D. Malioutov, M. Cetin, A. S. Willsky, A sparse signal reconstruction perspective for source localization with sensor arrays. IEEE Trans. Sig. Process. 53(8), 3010-3022 (2005)

11. X. Yang, C. C. Ko, Z. Zheng, Direction-of-arrival estimation of incoherently distributed sources using bayesian compressive sensing. IET Radar Sonar Navig. 10(6), 1057-1064 (2016)

12. Y. Shi, X. P. Mao, C. Zhao, Y. T. Liu, Underdetermined doa estimation for wideband signals via joint sparse signal reconstruction. IEEE Sig. Process. Lett. 26(10), 1541-1545 (2019)

13. J. Fuchs, in 2000 IEEE International Conference on Acoustics, Speech, and Signal Processing. Proceedings (Cat. No.00CH37100), On the application of the global matched filter to doa estimation with uniform circular arrays, vol. 5 (IEEE, Piscataway, 2000), pp. 3089-3092

14. Z. Liu, Z. Huang, Y. Zhou, J. Liu, Direction-of-arrival estimation of noncircular signals via sparse representation. IEEE Trans. Aerosp. Electron. Syst. 48(3), 2690-2698 (2012)

15. J. Cai, B. Wu, P. Li, W. Liu, in 2017 IEEE International Conference on Signal Processing, Communications and Computing (ICSPCC), A sparse representation based doa estimation algorithm for a mixture of circular and noncircular signals using sparse arrays (IEEE, Piscataway, 2017), pp. 1-5

16. M. Mañosas-Caballú, A. L. Swindlehurst, G. Seco-Granados, Power-based capon beamforming: Avoiding the cancellation effects of gnss multipath. Sig. Process. 180, 107891 (2021). https://doi.org/10.1016/j.sigpro.2020.107891

17. R. Schmidt, Multiple emitter location and signal parameter estimation. IEEE Trans. Antennas Propag. 34(3), 276-280 (1986)

18. F. G. Yan, L. Shuai, J. Wang, J. Shi, M. Jin, Real-valued root-music for doa estimation with reduced-dimension evd/svd computation. Sig. Process. 152, 1-12 (2018)

19. H. Fu, S. Abeywickrama, C. Yuen, M. Zhang, A robust phase-ambiguity-immune doa estimation scheme for antenna array. IEEE Trans. Veh. Technol. 68(7), 6686-6696 (2019)

20. C. P. Mathews, M. D. Zoltowski, Eigenstructure techniques for 2-d angle estimation with uniform circular arrays. IEEE Trans. Sig. Process. 42(9), 2395-2407 (1994)

21. Z. Zheng, W. Wang, H. Meng, H. C. So, H. Zhang, Efficient beamspace-based algorithm for two-dimensional doa estimation of incoherently distributed sources in massive mimo systems. IEEE Trans. Veh. Technol. 67(12), 11776-11789 (2018)

22. F. Belloni, A. Richter, V. Koivunen, Doa estimation via manifold separation for arbitrary array structures. IEEE Trans. Sig. Process. 55(10), 4800-4810 (2007) 
23. D. Liu, Y. Zhao, C. Cao, X. Pang, A novel reduced-dimensional beamspace unitary esprit algorithm for monostatic mimo radar. Dig. Sig. Process. 114, 103027 (2021). https://doi.org/10.1016/j.dsp.2021.103027

24. M. Y. Cao, L. Huang, C. Q., J. Y. Xue, H. C. So, Underdetermined doa estimation of quasi-stationary signals via khatri-rao structure for uniform circular array. Sig. Process. 106, 41-48 (2015)

25. S. F. Cotter, B. D. Rao, K. Engan, K. Kreutz-Delgado, Sparse solutions to linear inverse problems with multiple measurement vectors. IEEE Trans. Sig. Process. 53(7), 2477-2488 (2005)

26. J. D. Blanchard, C. Leedy, Y. Wu, On rank awareness, thresholding, and music for joint sparse recovery. Appl. Comput. Harmon. Anal. 48(1), 482-495 (2020)

27. F. Belloni, V. Koivunen, Beamspace transform for uca: Error analysis and bias reduction. IEEE Trans. Sig. Process. 54(8), 3078-3089 (2006)

28. W. Ma, T. Hsieh, C. Chi, Doa estimation of quasi-stationary signals with less sensors than sources and unknown spatial noise covariance: A khatri-rao subspace approach. IEEE Trans. Sig. Process. 58(4), 2168-2180 (2010)

29. E. J. Candes, T. Tao, Near-optimal signal recovery from random projections: Universal encoding strategies? IEEE Trans. Inf. Theory. 52(12), 5406-5425 (2006)

30. H. Abeida, J. P. Delmas, Efficiency of subspace-based estimators for elliptical symmetric distributions. Sig. Process. 174, 107644 (2020). https://doi.org/10.1016/j.sigpro.2020.107644

\section{Publisher's Note}

Springer Nature remains neutral with regard to jurisdictional claims in published maps and institutional affiliations.

\section{Submit your manuscript to a SpringerOpen ${ }^{\circ}$ journal and benefit from:}

- Convenient online submission

- Rigorous peer review

- Open access: articles freely available online

- High visibility within the field

- Retaining the copyright to your article

Submit your next manuscript at $\gg$ springeropen.com 